\title{
Exposição ao tabagismo entre escolares no Brasil
}

\author{
Smoking exposure among school children in Brazil
}

Sandhi M aria Barreto ${ }^{1}$

Luana Giatti ${ }^{1}$

Leticia Casado ${ }^{2}$

Lenildo de M oura ${ }^{3}$

Claudio Crespo ${ }^{4}$

Deborah Carvalho $\mathrm{M}$ alta ${ }^{3}$
${ }^{1}$ Faculdadede M edicina, UniversidadeFederal de Minas Gerais. Avenida Alfredo Balena 100, sala 814. 30320-050 Belo Horizonte M G. sbarreto@medicina.ufmg.br ${ }^{2}$ Instituto Nacional do Câncer.

${ }^{3}$ Coordenação Geral de Vigilância de Doençase Agravos Não

Transmissíveis, Secretaria deVigilância em Saúde Ministério da Saúde. ${ }^{4}$ Instituto Brasileiro de Geografia e Estatística.
Abstract The article describes the prevalence of tobacco exposure among adolescents at the $\mathrm{Na}$ tional Adolescent School-based Health Survey (PeN SE) and investigates socio-demographic and behavioral factors associated with smoking. The profile of a current smoker was defined as reporting having smoked at least one cigarette in the previous 30 days. The socio-demographic characteristics studied were age, sex, race/skin color, mother education, household assets index and school (public or private). Risk and protective behaviors included were alcohol and drug experimentation, sexual intercourse, consumption of at least one glass of alcohol in the past 30 days and perform or be willing to perform physical activity on most days of the weeks. Prevalence of current smoker was $6.3 \%(95 \% \mathrm{Cl}: 5,87-6,74)$ and was positively associated with older age, lower education of the mother, brown skin color, study in public school and presence of all therisk and protective behaviors studied. $\mathrm{O} n$ the multivariate analysis, smoking remained associated with age and risk behaviors. To perform or be willing to perform physical activity were inversely related to smoking. The coexistence of risk behaviors is also present in adolescence, suggesting that health promotion polices to the adolescence might have a broader impact, including on tobacco use. Key words Smoking, School health, Adolescence, $\mathrm{H}$ ealth risk behavior
Resumo 0 objetivo deste trabalho é descrever a exposição ao tabagismo de participantes da Pesquisa N acional deSaúdedo Escolar (PeNSE) eidentificar fatores associadosa ele. Para seapresentar 0 perfil detabagismo regular, era preciso ter fumado cigarro pelo menosum dia nos 30 dias anterioresà pesquisa. As características estudadas foram idade, sexo, raça/cor, escolaridade da mãe, índice de bens do domicílio e dependência administrativa da escola. Os comportamentos de risco e proteção incluídos foram experimentar álcool ou drogas, ter relação sexual, consumo de $\geq 1$ copo de bebida alcoólica nos últimos 30 dias e realizar/ter interesse em realizar atividade física na maioria dos dias da semana. A prevalência de fumante regular foi 6,3\% (IC95\%:5,87-6,74), sendo estatisticamente maior nos mais velhos, de cor parda, cujas mães têm menor escolaridade, estudantes em escolas públicas e apresentando os comportamentos estudados. $\mathrm{Na}$ análise multivariável, o tabagismo permaneceu associado à idadeea comportamentos de risco. A chance de fumar também foi menor em quem não faz nem gostaria de fazer atividadefísica. Conclusão: comportamentos de risco para a saúde coexistem também na adolescência, sugerindo que políticas de promoção da saúdena adolescência provavelmente terão impacto amplo, incluindo o problema do tabagismo.

Palavras-chave Tabagismo, Saúde escolar, Adolescência, Comportamentos de risco 
Introdução

Os adolescentes são um dos maiores grupos populacionais em todo o mundo, somando cerca de 1,197 bilhão dejovens entre 10 e 19 anos em $2010^{1}$. Mudanças biológicas, culturais e sociais ocorridas na adolescência tornam esse período da vida especialmentesuscetível a experimentações emudanças comportamentais, tais como tabaco, álcool, drogas, sexo e dieta, com impacto sobre a saúde corrente e ao longo do curso de vida².

Estudos em diversos países, incluindo o Brasil, mostram que a exposição ao tabagismo na adolescênciaéum importante desafio para a saúde pública. No Reino Unido, aos 12 anos, 10\% dos adolescentes relataram ter fumado cigarro na semana anterior - aos 16 anos, esse percentual cresceu para $25 \%^{3}$. Nos Estados Unidos, em 2002, $18 \%$ dos adolescentes da $10^{a}$ série escolar relataram haver fumado pelo menos uma vez nos últimos trinta dias ${ }^{4}$. No Brasil, estudo no sul do país entre escolares de 13 a 15 anos encontrou uma prevalência de tabagismo nos últimos trinta dias variando de $11 \%$ em Florianópolis (SC) a $18 \%$ em Porto Alegre (RS) 5 .

A exposição ao tabagismo na adolescência tem inúmeras eimportantes implicações para o bemestar e a saúde do adolescente, a curto, médio e longo prazos ${ }^{4}$. Estudo de coortenos Estados Unidos mostrou que metade dos adolescentes que experimentaram tabaco fumava diariamenteum ano depois ${ }^{6}$. A dependência da nicotina também podeter início na adolescência e não dependedo uso diário de tabaco. Dados longitudinais coletadosanualmentede 1.246 escolares (2002-2006), utilizando 11 entrevistas individuais, mostraram que o uso de cigarro irregular (não diário) pode disparar a dependência da nicotina. Sintomas de dependência precoce promovem uma escalada na frequência do uso de tabaco; os que usam mais frequentemente tendem a apresentar mais sintomas de dependência ${ }^{7}$. De maneira geral, a revisão de resultados de pesquisas na área indica que de cada cinco adolescentes que usam cigarro, um a três são dependentes de nicotinå.

Além dos problemas durante a adolescência, há indicações de que o uso de tabaco na adolescência está associado ao uso na vida adulta9. Peto et al. ${ }^{10}$ mostraram que indivíduos que começam a fumar em idade igual ou inferior a 15 anos tem duas vezes mais risco de câncer de pulmão quando comparados aos que iniciam aos vinte anos ou mais. Análises de curso de vida mostram que circunstâncias adversas na infância, incluindo 0 tabagismo, estão associadas a uma pior autoavaliação de saúde na vida adulta ${ }^{11}$. Além disso, estudo finlandês mostrou que comportamentos de risco em saúde na adolescência predizem menor nível educacional na vida adulta, contribuindo para aumentar as desigualdades em saúde ${ }^{12}$.

0 presente estudo tem por objetivo descrever a exposição ao tabagismo entre escolares participantes da Pesquisa Nacional de Saúde do Escolar (PeNSE) e identificar fatores sociodemográficos, além de comportamentos de risco e proteção associados ao tabagismo, com o intuito de orientar políticas públicas de promoção da saúde e redução da exposição ao tabagismo na adolescência.

\section{Material eM étodos}

Este estudo utilizou dados da primeira Pesquisa Nacional de Saúde do Escolar realizada em escolares do $9^{\circ}$ ano (antiga $8^{a}$ série) do ensino fundamental de escolas públicas e privadas das 26 capitais dos estados brasileiros e do Distrito Federal, nos meses de maio a junho de 2009.

A PeNSE utilizou uma amostra probabilística complexa em dois estágios, em que primeiro foram selecionadas as escolas e em seguida as turmas de $9^{\circ}$ ano. As escolas formaram estratos por capitais dos estados e do Distrito Federal, totalizando 27 estratos geográficos. As escolas em cada estrato foram agrupadas segundo sua dependência administrativa (particular ou pública), e o tamanho da amostra foi alocado proporcionalmente em cada um desses grupos.

A pesquisa utilizou questionário estruturado autoaplicável administrado nas salas de aulas e preenchido pelos alunos em um computador de mão (palmtop), o Personal Digital Assistant (PDA). 0 questionário contém módulos sobre diversos aspectos da vida dos escolares: características sociodemográficas, alimentação, imagem corporal, atividade física, tabagismo, consumo de álcool e outras drogas, saúde bucal, comportamento sexual, exposição à violência ea acidentes, percepção dos alunos sobre a família e apreciação geral do questionário.

A participação do aluno foi voluntária e havia a possibilidade de se deixar de responder a qualquer pergunta ou a todo o questionário. Todas as informações do aluno foram confidenciais e não identificadas, assim como as da escola. A metodologia do inquérito foi descrita por Malta et al. ${ }^{13}$. 
Variáveis

No presenteestudo, foram utilizadas as seguintes variáveis para descrever a exposição ao tabagismo: experimentação de cigarros al guma vez na vida, obtida pela pergunta "Alguma vez na vida você já fumou cigarro, mesmo uma ou duas tragadas?", categorizada em não e sim; a idade de experimentação, cuja pergunta utilizada foi “Queidade você tinha quando experimentou cigarro pela primeiravez?"; eo tabagismo regular definido como relato de ter fumado cigarros pelo menos um dia nos últimos trinta dias anteriores à realização da pesquisa, obtida pela pergunta "N os últimos trinta dias, em quantos dias você fumou cigarros?".

Para o estudo da associação entre fatores individuais e contextuais e o tabagismo no último mês, a variável resposta utilizada foi tabagismo regular. As variáveis explicativas foram agrupadas por afinidade em dois blocos de covariáveis, descritos a seguir:

1) Características sociodemográficas dos escolares: sexo (masculino efeminino), idadeem anos, raça/cor (branca, preta, parda, amarela, indígena), escolaridade materna (nível universitário completo, universitário incompleto, ensino mé dio incompleto, ensino fundamental incompleto, não estudou, não sabe informar), índice de bens do domicílio, que variou de 0 a 1 efoi agrupado em tercis, e dependência administrativa da escola (pública ou privada). Para o cálculo do índice, foram considerados todos os bens com prevalência menor que $70 \%$ no conjunto da amostra (tel efone fixo, máquina de lavar roupas, microcomputador com internet, carro emoto), atribuindo-se pesos à presença de cada um que correspondeu ao complemento da frequência relativa na amostra estudada ${ }^{14}$.

2) Comportamentos de risco/proteção do escolar: experimentação de bebida alcoólica al guma vez na vida (não, sim); relato de consumo de pelo menos um copo de bebida al coólica nos últimos trinta dias (não, sim); relato de relação sexual alguma vez na vida (não, sim); uso dedrogas - maconha, cocaína, crack, cola, loló, lançaperfume, ecstasy ou outra - alguma vez na vida (não, sim); se faz ou faria atividade física na maioria dos dias da semana (se já faz atividade física na maioria dos dias da semana, faria atividade física na maioria dos dias da semana, não faria), por meio da pergunta"Sevocêtivesseoportunidade de fazer atividade física na maioria dos dias da semana, qual seria sua atitude?".

A PeNSE foi registrada na Comissão Nacional de Ética em Pesquisa (Conep), que se mani- festou pela aprovação do projeto de pesquisa proposto.

\section{Análise}

Inicialmente, foi feita a descrição das variáveis que caracterizam a exposição ao tabagismo por sexo eidade: experimentação pelo menosuma vez na vida, idade de experimentação e tabagismo regular, segundo sexo e idade.

A associação entre tabagismo regular e as variáveis explicativas independentes foi medida por meio do teste do qui-quadrado de Pearson, com nível de significância de 0,05. Em seguida, utilizando-se regressão logística, procedeu-se à análise univariada para se obter a razão de odds (OR) e seu intervalo de confiança de $95 \%$. Por último, realizou-se a análise multivariável por blocos de variáveis explicativas, utilizando as covariáveis que apresentaram associação com tabagismo regular ao nível $p<0,20$ na análise univariada. A idadefoi acrescida à análise multivariável dos fatores comportamentais de risco e proteção.

Para corrigir as diferentes probabilidades de seleção de cada escolar, foram utilizados pesos na estimativa das proporções. A análise foi feita no software Stata (versão 9.2), utilizando o procedimento "svy" (com fatores de ponderação) adequado para análises de dados obtidos por plano amostral complexo.

\section{Resultados}

Entre os 60.973 escolares que participaram da PeN SE, 981 foram excluídos do presente estudo porque não responderam à pergunta sobre o uso de cigarro nos últimos trinta dias. Dos 59.992 alunos estudados, $52,6 \%$ eram do sexo feminino; $23,8 \%$ tinham 13 anos; $47,1 \%, 14$ anos; e $18,2 \%, 15$ anos de idade.

Experimentação de cigarro pelo menos uma vez na vida foi informada por $24 \%$ (IC95\%:23,29-25,05) dos escolares; essa proporção aumentou com a idade, passando de $16 \%$ (IC95\%:15,03-17,34) entre os escolares de até 13 anos a $41 \%$ (IC95\%:39,50-43,11) entre os que tinham 16 anos ou mais, mas não houve diferença entre sexos. A prevalência de fumante regular foi de 6,3\% (IC95\%:5,87-6,74); também aumentou com a idade, chegando a $14,4 \%$ (IC95\%:12,9-16,0) entre os que tinham 16 anos ou mais e não variou entre meninos e meninas. Entre os que fumaram cigarro pelo menos uma vez na vida, 49,3\% (IC95\%:47,67-50,93) o fize- 
ram até os 12 anos de idade, sendo que os meninos tendem a experimentar em idade mais precoce do que as meninas.

A Tabela 1 apresenta a distribuição dos escolares segundo o relato de tabagismo regular e as características sociodemográficas dos escolares. Entre as características sociodemográficas, dife renças significativas entre as prevalências de escolares que relatam tabagismo regular foram observadas somente em relação à idade deles, à escolaridade materna e à dependência administrativa da escola.

A prevalência de adolescentes que relataram ter fumado nos últimos trinta dias foi mais elevada entre aqueles que experimentaram álcool, tiveram relação sexual e fizeram uso de drogas alguma vez na vida e os que consumiram pelo menos uma dose de álcool nos últimos trinta dias. Já os adolescentes que faziam atividade físi- ca na mai oria dos dias da semana, ou que fariam caso tivessem oportunidade, apresentaram menores prevalências de tabagismo (Tabela 2).

$\mathrm{Na}$ análise multivariável das características sociodemográficas, permaneceram associadas ao tabagismo regular apenas a idade $(O R=1,40$, IC95\%:1,15-1,69- 14 anos; O R =3,01, I C 95\%:2,403,79-15 anos; O R =4,69 IC 95\%:3,78-5,81- 16 anos ou mais) e a raça/cor parda ( $O R=0,85$; IC $95 \%: 0,75-0,96)$. A escolaridade materna e a dependência administrativa da escola não foram estatisticamente associadas ao tabagismo regular (Tabela 3).

$\mathrm{Na}$ Tabela 3 são apresentados os resultados das análises multivariáveis após ajuste pelas variáveis do mesmo bloco e da idade. Todas as variáveis comportamentais de risco (ter experimentado álcool, drogas e ter feito sexo pelo menos uma vez na vida e ter consumido pelo menos

Tabela 1. Prevalência de tabagismo regular* segundo características sociodemográficas dos escolares das capitais dos estados brasileiros e do Distrito Federal. PeN SE, 2009.

\begin{tabular}{|c|c|c|c|c|}
\hline Características sociodemográficas & $\begin{array}{l}\text { Prevalência \% } \\
\text { (IC95\%) }\end{array}$ & OR & IC95\% & Valor de $p$ \\
\hline \multicolumn{5}{|l|}{ Sexo } \\
\hline M asculino & $6,4(5,9-6,9)$ & 1,00 & & \multirow[t]{2}{*}{$p=0,789$} \\
\hline Feminino & $6,3(5,7-6,9)$ & 0,98 & $(0,86-1,12)$ & \\
\hline \multicolumn{5}{|l|}{ I dade (anos) } \\
\hline$<13$ & $3,5(2,9-4,2)$ & 1,00 & & \multirow[t]{4}{*}{$p<0,001$} \\
\hline 14 & $4,8(0,4-0,5)$ & 1,40 & $(1,15-1,71)$ & \\
\hline 15 & $9,7(8,7-10,7)$ & 2,99 & $(2,37-3,76)$ & \\
\hline 16 ou mais & $14,4(12,9-16,0)$ & 4,69 & $(3,76-5,85)$ & \\
\hline \multicolumn{5}{|l|}{ Raça/cor } \\
\hline Branca & $6,3(5,7-6,9)$ & 1,00 & & \multirow[t]{5}{*}{$p=0,101$} \\
\hline Preta & $7,2(6,2-8,2)$ & 1,16 & $(0,97-1,38)$ & \\
\hline Parda & $6,0(5,4-6,6)$ & 0,95 & $(0,83-1,08)$ & \\
\hline Amarela & $6,1(4,5-6,6)$ & 0,97 & $(0,70-1,35)$ & \\
\hline Indígena & $7,7(6,3-9,3)$ & 1,25 & $(1,01-1,54)$ & \\
\hline \multicolumn{5}{|l|}{ Escolaridade materna } \\
\hline Universitário completo & $5,6(4,8-6,6)$ & 1,00 & & \multirow[t]{6}{*}{$p=0,001$} \\
\hline Universitário incompleto & $6,0(5,3-6,7)$ & 1,07 & $(0,88-1,30)$ & \\
\hline Ensino médio incompleto & $6,7(5,7-7,8)$ & 1,21 & $(0,97-1,50)$ & \\
\hline Ensino fundamental incompleto & $6,7(5,9-7,5)$ & 1,20 & $(0,98-1,48)$ & \\
\hline Não estudou & $10,4(8,3-10,1)$ & 1,96 & $(1,46-2,65)$ & \\
\hline $\mathrm{N}$ ão sabe informar & $5,9(5,0-7,0)$ & 1,05 & $(0,83-1,34)$ & \\
\hline \multicolumn{5}{|l|}{ Índice de bens } \\
\hline $1^{\circ}$ tercil (menor) & $6,3(5,7-7,0)$ & 1,00 & & \multirow[t]{3}{*}{$p=0,735$} \\
\hline $2^{\circ}$ tercil & $6,1(5,5-6,7)$ & 0,96 & $(0,83-1,12)$ & \\
\hline $3^{\circ}$ tercil (maior) & $6,4(5,7-7,2)$ & 1,02 & $(0,87-1,19)$ & \\
\hline \multicolumn{5}{|l|}{ Domínio administrativo da escola } \\
\hline Privada & $6,6(6,1-7,1)$ & 1,00 & & \multirow[t]{2}{*}{$p=0,001$} \\
\hline Pública & $5,3(4,6-6,1)$ & 0,80 & $(0,67-0,95)$ & \\
\hline
\end{tabular}

* Relato de ter fumado cigarros pelo menos um dia nos últimos trinta dias anteriores à realização da pesquisa. 
Tabela 2. Prevalência de tabagismo regular" segundo comportamentos de risco e proteção dos escolares das capitais dos estados brasileiros e do Distrito Federal. PeN SE, 2009.

\begin{tabular}{|c|c|c|c|c|}
\hline Comportamentos de risco/proteção & $\begin{array}{l}\text { Prevalência \% } \\
\text { (IC95\%) }\end{array}$ & OR & IC95\% & Valor de $p$ \\
\hline \multicolumn{5}{|c|}{ Experimentação de bebida alcoólica na vida } \\
\hline Não & $4,1(2,9-5,7)$ & 1,00 & & $p<0,001$ \\
\hline Sim & $8,7(8,1-9,3)$ & 23,10 & $(16,46-32,41)$ & \\
\hline \multicolumn{5}{|c|}{ Consumo de pelo menos um copo de bebida } \\
\hline $\begin{array}{l}\text { nosúltimos trinta dias } \\
\text { Não }\end{array}$ & $\begin{array}{r}1,6(1,4-1,8) \\
18.8(17-6-20.1)\end{array}$ & $\begin{array}{r}1,00 \\
14.16\end{array}$ & $(12,22-16,42)$ & $p<0,001$ \\
\hline \multicolumn{5}{|l|}{ Sim } \\
\hline Experimentação de drogas navida & $3,4(3,1-3,7)$ & 1,00 & & $p<0,001$ \\
\hline $\begin{array}{l}\text { Não } \\
\text { Sim }\end{array}$ & $36,9(34,5-39,5)$ & 16,69 & $(14,39-19,36)$ & \\
\hline Relação sexual na vida & $2,8(2,5-3,1)$ & 1,00 & & $p<0,001$ \\
\hline Não & $15,3(14,3-16,4)$ & 6,33 & $(5,54-7,22)$ & \\
\hline Sim & & & & \\
\hline \multicolumn{5}{|l|}{ Faz ou faria atividade física na maioria } \\
\hline Não faria & $5,9(5,4-6,5)$ & 0,65 & $(0,54-0,78)$ & \\
\hline $\begin{array}{l}\text { Faria sepudesse } \\
\text { láfaz }\end{array}$ & $6,3(5,6-7,0)$ & 0,69 & $(0,58-0,81)$ & \\
\hline
\end{tabular}

* Relato de ter fumado cigarros pelo menos um dia nos últimos trinta dias anteriores à realização da pesquisa.

uma dose de bebida alcoólica nos últimos trinta dias) aumentaram as chances de tabagismo regular. Realizar ou ter a disposição de realizar atividades físicas na maioria dos dias da semana esteve associado à menor chance de fumar cigarros (Tabela 4).

\section{Discussão}

Neste estudo identificamos que quase um quarto dos escolares participantes da PeNSE experimentaram cigarro pelo menos uma vez na vida, e que metade desses escolares tinha doze ou menos anos de idade quando experimentaram pela primeira vez. A prevalência de uso regular de cigarro, definida como o uso em pelo menos um dia nos últimos trinta dias, foi menor quea relatada para vários países ocidentais e não diferiu entre meninos e meninas. Os achados do presente estudo corroboram a associação positiva entre tabagismo e outros comportamentos de risco para a saúde, e indicam que a prática esportiva, presente ou desejada, está associada negativamente ao tabagismo.

A prevalência de experimentação observada na PeNSE foi bem inferior à média encontrada pela pesquisa sobre comportamentos em saúde
Tabela 3. Associação entre tabagismo regular e características sociodemográficas entre escolares das capitais dos estados brasileiros e do Distrito Federal. PeN SE, 2009.

\begin{tabular}{lrr}
\hline Características sociodemográficas & $\mathrm{OR}^{*}(\mathrm{IC} 95 \%)$ \\
\hline Idade (anos) & & \\
$<13$ & 1,00 & \\
14 & 1,40 & $(1,15-1,69)$ \\
15 & 3,02 & $(2,40-3,80)$ \\
16 ou mais & 4,69 & $(3,77-5,83)$ \\
Raça/cor & & \\
$\quad$ Branca & 1,00 & \\
Preta & 0,91 & $(0,77-1,09)$ \\
Parda & 0,85 & $(0,75-0,96)$ \\
Amarela & 0,88 & $(0,63-1,23)$ \\
Indígena & 1,10 & $(0,88-1,36)$ \\
Escolaridade materna & & \\
Universitário completo & 1,00 & \\
Universitário incompleto & 0,99 & $(0,81-1,20)$ \\
Ensino médio incompleto & 1,03 & $(0,82-1,28)$ \\
Ensino fundamental incompleto & 0,92 & $(0,79-1,15)$ \\
Não estudou & 1,32 & $(0,96-1,81)$ \\
Não sabe informar & 0,88 & $(0,68-1,12)$ \\
Domínio administrativo da escola & & \\
Privada & 1,00 & \\
Pública & 1,01 & $(0,83-1,24)$ \\
& &
\end{tabular}

*OR ajustado por todos os fatores incluídos na tabela. 
Tabela 4. Associação entre tabagismo regular e comportamentos selecionados de risco e proteção à saúde entre escolares das capitais dos estados brasileiros e do Distrito Federal. PeN SE, 2009.

\begin{tabular}{|c|c|c|}
\hline $\begin{array}{l}\text { Comportamentos } \\
\text { de risco/proteção }\end{array}$ & \multicolumn{2}{|c|}{ OR* (IC95\%) } \\
\hline \multicolumn{3}{|l|}{$\begin{array}{l}\text { Experimentação de bebida } \\
\text { alcoólica na vida }\end{array}$} \\
\hline Não & 1,00 & \\
\hline Sim & 4,52 & $(2,99-6,83)$ \\
\hline \multicolumn{3}{|c|}{$\begin{array}{l}\text { Consumiu pelo menos um copo } \\
\text { de bebida alcoólica nos últimos }\end{array}$} \\
\hline trinta dias & 1,00 & \\
\hline Não & 5,72 & $(4,84-6,75)$ \\
\hline Sim & & \\
\hline \multicolumn{3}{|c|}{ Experimentação de drogas na vida* } \\
\hline Não & 1,00 & \\
\hline Sim & 6,86 & $(5,78-8,13)$ \\
\hline \multicolumn{3}{|l|}{ Relação sexual na vida } \\
\hline Não & 1,00 & \\
\hline $\mathrm{Sim}$ & 1,98 & $(1,70-2,31)$ \\
\hline \multicolumn{3}{|c|}{$\begin{array}{l}\text { Faz ou faria atividade física } \\
\text { na maioria dos dias da semana }\end{array}$} \\
\hline Não faria & 1,00 & \\
\hline Faria se pudesse & 0,75 & $(0,61-0,93)$ \\
\hline Já faz & 0,68 & $(0,56-0,82)$ \\
\hline
\end{tabular}

* OR ajustado por todos os fatores incluídos na tabela e pela idade.

realizada em 2001/2002 em escolares de 35 países europeus, com idades de 13 e 15 anos (43\% e $62 \%$, respectivamente) ${ }^{15}$. Pesquisa recentena Índia também revelou maior prevalência de expe rimentação em escolares da $6^{a}$ série, com idade média de 13 anos (32\%) ${ }^{16}$. Diferentemente da pesquisa da Organização Mundial da Saúde (OMS) na Europa, já citada, não encontramos diferença estatística na prevalência deexperimentação entre os gêneros. Entre os escolares europeus, a prevalência foi maior entre o sexo masculino na idade de 11 anos e maior entre o sexo feminino na idade de 15 anos, sugerindo que os meninos experimentam mais cedo.

A experimentação éum problema importante e parece estar associada à adição ao tabaco. Gilpin et al. estimaram que cerca da metade dos adolescentes que já experimentaram cigarro e apenas $9 \%$ dos que nunca fumaram correm risco de se tornarem fumantes ${ }^{17}$. Já a influência da idade de experimentação no tabagismo futuro aindaécontroversa. Breslau et al. verificaram que indivíduos que começavam a fumar antes de 14 anos não tinham maior probabilidade de fumar diariamente quando comparados aos que inicia- vam depois dessa idade ${ }^{18}$. Entretanto, outro estudo, após controle por fatores de confusão, verificou que a probabilidade de cessação é significativamente maior em pessoas que começam a fumar aos 14 anos, em comparação às que começam a fumar aos 13 anos ou menos ${ }^{19}$.

A prevalência de tabagismo regular encontrada na PeN SE também foi menor que a prevalência média observada na vigilância global de tabaco em jovens (GYTS), com a coordenação do Centro para Controles de Doenças (CDC) dos Estados Unidos, queinclui escolares com idades entre 13 e 15 anos de 151 países $^{20}$. Os resultados de 2000-2007 mostram que a prevalência de uso regular de tabaco, utilizando a mesma definição da PeNSE, foi de 9,5\%, variando de 4,9\% na região das Américas até 19\% na região europeia. A GYTS também não identificou diferença significativa na prevalência detabagismo regular entre os sexos em nenhuma dessas duas regiões. Considerando que a composição etária da PeN SE e da GYTS é similar, é improvável que a idade seja uma explicação para a menor prevalência de tabagismo regular encontrada no país.

Revisão sistemática de estudos na América Latina mostra que a prevalência de tabagismo regular na PeNSE foi cerca da metade dos níveis encontrados por outros estudos brasileiros real izados com escolares que usaram a mesma definição, e cinco vezes menor do que a observada no Chile ${ }^{21}$. É interessante notar que a diferença entre sexos também não foi identificada na maioria dos estudos realizados no Brasil incluídos na referida revisão. M esmo considerando que não dispomos de dados que permitam traçar a ten dência da prevalência de tabagismo entre adolescentes no país, a comparação com dados pontuais de estudos anteriores com escolares sugere queda na prevalência de tabagismo entre escolares. Tal observação está em compasso com a tendência de queda do tabagismo entreadultos no paíse, assim como esta, é possível que reflita o impacto das ações antitabágicas adotadas no Brasil.

Entre as características sociodemográficas analisadas, além da raça/cor parda, idade foi o único fator positiva e independentemente associado ao tabagismo regular em escolares. Este achado é equival ente à grande mai oria dos estudos realizados no mundo. 0 Vigescola, por exemplo, não identificou diferença estatística na prevalência entre os sexos, após ajustar por alguns fatores de confusão, em nenhum dos estados do sul do país ${ }^{5}$. Com relação aos indicadores sociais, nem a escolaridade materna nem o índice de bens apresentaram associação com o tabagismo regular na presente análise. Estudo prospec- 
tivo de 877 estudantes canadenses com média etária de 12,7 anos não identificou influência da escolaridade dos pais sobre o risco de experimentar cigarro ou de progredir para tabagismo diário entreos estudantes quehaviam experimentado ${ }^{22}$. Os resultados de estudos que investigaram a influência de fatores socioeconômicos sobre a prevalência de tabagismo regular são inconsistentes, mas a maioria dos estudos não encontra associação estatisticamente significante 4 . Diversos estudos, inclusive no Brasil, sugerem que há uma atenuação das desigualdades sociais em saúde no período da adolescência, mas não há explicações consen suais para tal observação ${ }^{23}$.

A coexistência de comportamentos de risco para a saúde está presente também na adolescência. Fumar está consistentemente associado a vários outros comportamentos de risco, entre os quais o uso de drogas eálcoo|24,25. Tal associação sugere que fatores contextuais comuns, incluindo família, colegas e escola, concorrem para aumentar o risco de experimentação ou iniciação precoce desses comportamentos.

A associação protetora entre tabagismo e a prática de esportestambém foi descrita na literatura e provavelmente reflete os cenários e características que facilitam o tabagismo como distintos daqueles que promovem a saúde do adolescente. Neste estudo, utilizamos uma pergunta subjetiva para avaliar a associação entre esporte e tabagismo. 0 interesse de usar essa pergunta foi caracterizar que mesmo entre aqueles que não praticam regularmente existeum percentual que não o faz por falta de oportunidade, mas que se diferencia dos escolares que não praticam nem desejam praticar esportes, grupo este que apresenta a maior prevalência de tabagismo regular. 0 engajamento em atividades esportivas é uma fonte de prazer e contribui para o desenvolvimento físico, emocional e social do adolescente ${ }^{26}$. A associação encontrada reforça a importância de se ampliarem as oportunidades de práticas esportivas para o jovem no país como uma ação promotora da saúde no sentido mais amplo, e também como preventiva para o tabagismo e outros comportamentos de risco.

Apesar de a PeN SE ser a maior pesquisa com escolares no país, com mais de 60 mil participantes, ela foi realizada apenas nas capitais dos estados e no Distrito Federal. Portanto, a rigor, a prevalência média estimada neste estudo não representa a população escolar da ga série do ensino fundamental do país. É possível que a distribuição das características dos estudantes ea prevalência detabagismo encontrada sejam um pouco diferentes nas cidades do interior dos estados, especialmentenas cidades de pequeno porte. Porém, como a grande maioria da nossa população resideem municípios demédio egrandeportes, é possível quea realidade examinada reflita a grandemai oria dos estudantes de ga sérieno país.

Este estudo utilizou um conjunto de indicadores aferidos na PeN SE para analisar os fatores sociodemográficos e comportamentais associados ao tabagismo entre escolares. As informações foram aferidas por entrevista autoaplicada e podem conter erros de aferição por sub-relato ou mesmo por diferentes níveis de dificuldade de compreensão das perguntas pel os alunos participantes da pesquisa. A escol ha dealunos da ${ }^{a}$ série visou minimizar ao máximo a influência de possíveis diferenças na habilidade de leitura dos alunos incluídos na amostra. Também foi precedida por estudos piloto que testaram a compreensão do questionário; quanto ao potencial de sub-relato, é possível, como atesta estudo realizado no sul do país ${ }^{27}$. Entretanto, esse é um método não invasivo, que respeita a confidencialidade da informação e é utilizado em todas as pesquisas internacionais. Ao mesmo tempo, essemétodo permite real izar uma pesquisa em larga escala como a PeN SE. Além disso, acreditamos que o uso do Personal Digital Assistant (PDA) criaum ambiente de maior privacidade para as respostas, o que deve favorecer a confiabilidade delas.

\section{Conclusão}

Os resultados da análise reforçam a importância do tabagismo na adolescência e sua relação positiva com outros fatores de risco para a saúde. Políticas públicas de promoção da saúde e prevenção do tabagismo em adolescentes devem levar em conta que comportamentos de risco para a saúde tendem a não ocorrer isoladamente e que melhorias no ambienteem quea criança vive equeela frequenta trarão, certamente, benefícios a múltiplos aspectos do bem-estar e da saúde dos adolescentes.

\section{Colaboradores}

SM Barreto e L Giatti analisaram os dados e redigiram a primeira versão do artigo; L Casado, C Crespo, L M oura e D M alta participaram da idealização do artigo e contribuíram para a sua versão final. 


\section{Referências}

1. US Census Bureau. International Data Base (IDB) [site da Internet] [acessado 2010 jul 29]. Disponível em: http://www.census.gov/cgi-bin/broker

2. Richter LM. Studying adolescence. Science 2006; 312(5782):1902-1905.

3. Baker T, Brandon T, Chassin L. Motivational influences on cigarette smoking. Annual Review of Psychology 2004; 55:463-491.

4. Turner L, Mermelstein R, Flay B. Individual and contextual influences on adolescent smoking. Ann N Y Acad Sci 2004; 1021:175-197.

5. Hallal AL, Gotlieb SL, Almeida LM, Casado L. Prevalence and risk factors associated with smoking among school children, Southern Brazil. Rev Saude Publica 2009; 43(5):779-788.

6. Difranza JR, Rigotti NA, M cNeill AD, Ockene JK, Savageau JA, St Cyr D, Coleman M. Initial symptoms of nicotine dependence in adolescents. Tob Control 2000; 9(3):313-319.

7. Doubeni CA, Reed G, Difranza JR. Early course of nicotine dependence in adolescent smokers. Pediatrics. 2010. [In press].

8. Colby SM, Tiffany ST, Shiffman S, Niaura RS. Are adolescent smokers dependent on nicotine? A review of the evidence. Drug Alcohol Depend 2000; 59(Suppl 1):S83-95.

9. Johnson CC, Webber LS, Myers L, Boris NW, Berenson GS. Co-use of alcohol and tobacco among ninth-graders in Louisiana. Prev Chronic Dis 2009; 6(3):A85.

10. Peto R, Darby S, Deo H, Silcocks P, Whitley E, Doll R. Smoking, smoking cessation, and lung cancer in the UK since 1950: combination of national statistics with two case-control studies. British Medical Journal 2000; 321:323-329.

11. Kestilä $L, M$ artelin $T$, Rahkonen $O, H$ ärkänen $T$, Koskinen S. The contribution of childhood circumstances, current circumstances and health behaviour to educational health differences in early adulthood. BM C Public Health 2009; 9:164.

12. Koivusilta L, Rimpelä A, Vikat A. Health behaviours and health in adolescence as predictors of educational level in adulthood: a follow-up study from Finland. Soc Sci M ed 2003; 57(4):577-593.

13. M alta DC, Sardinha LM V, M endes I, Barreto SM, Giatti L, Castro IRR, M oura L, Dias AJR, Crespo C. Prevalência de fatores de risco e proteção de doenças crônicas não transmissíveis em adolescentes: resultados da Pesquisa Nacional de Saúde do EscoIar (PeN SE), Brasil, 2009. Cien Saude Colet 2010; 15 (Supl.2):3009-3019.

14. Szwarcwald $C L$, Souza Júnior PRB, Esteves MAP, Damacena GN, Viacava F. Socio-demographic determinants of self-rated health in Brazil. Cad Saude Publica 2005; 21(Supl.1):S54-64.

15. Currie C, Roberts C, M organ A, Smith R, Settertobulte W, Samdal O, Rasmussen VB, editors. Young people's health in context: $\mathrm{H}$ ealth Behaviour in SchoolAged Children (HBSC) study: international report from the 2001/2002 survey. Copenhagen: WHO Regional Office for Europe; 2004.
16. Reddy KS, Perry CL, Stigler M H, Arora M. Differences in tobacco use among young people in urban India by sex, socioeconomic status, age, and school grade: assessment of baseline survey data. Lancet 2006; 367(9510):589-594.

17. Gilpin EA, White VM, Pierce JP. What fraction of young adults are at risk for future smoking, and who are they? Nicotine Tob Res 2005; 7(5):747-759.

18. Breslau N, Fenn N, Peterson EL. Early smoking initiation and nicotine dependence in a cohort of young adults. Drug Alcohol Depend 1993; 33(2):129-137.

19. Breslau N, Peterson EL. Smoking cessation in young adults: age at initiation of cigarette smoking and other suspected influences. Am J Public H ealth 1996; 86(2):214-220.

20. Warren CW, Jones NR, Peruga A, Chauvin J, Baptiste JP, Costa de Silva V, el Awa F, Tsouros A, Rahman K, Fishburn B, Bettcher DW, Asma S. Centers for Disease Control and Prevention (CDC). Global Youth Tobacco Surveillance, 2000-2007. M M WR Surveill Summ 2008; 57(1):1-28.

21. $M$ alcon M C, M enezes $A M, M$ aia M F, Chatkin $M$, Victora CG. Prevalence of and risk factors for cigarette smoking among adolescents in South America: a systematic literature review. Rev Panam Salud Publica 2003; 13(4):222-228.

22. O'Loughlin J, Karp I, Koulis T, Paradis G, Difranza J. Determinants of first puff and daily cigarette smoking in adolescents. Am J Epidemiol 2009; 170(5):585-597.

23. Barreto SM, Giatti L, M artinez-Hernaez A. Contextual and family factors associated with negative assessment of children's health. Eur J Public Health 2010. [In press].

24. Leatherdale ST, Hammond D, Ahmed R. Alcohol, marijuana, and tobacco use patterns among youth in Canada. Cancer Causes Control 2008; 19(4):361-369.

25. Faeh D, Viswanathan B, Chiolero A, Warren W, Bovet P. Clustering of smoking, alcohol drinking and cannabis use in adolescents in a rapidly developing country. BMC Public Health 2006; 6:169.

26. Strong WB, M alina RM, Blimkie CJ, Daniels SR, Dishman RK, Gutin B, Hergenroeder AC, Must A, Nixon PA, Pivarnik JM, Rowland T, Trost S, Trudeau F. Evidence based physical activity for school-age youth. J Pediatr 2005; 146(6):732-737.

27. $M$ alcon $M C, M$ enezes $A M$, Assunção $M C$, N eutzling M B, Hallal PC. Agreement between self-reported smoking and cotinine concentration in adolescents: a validation study in Brazil. J Adolesc Health 2008; 43(3):226-230.

Artigo apresentado em 30/05/2010

A provado em 26/07/2010

Versão final apresentada em 11/08/2010 